\title{
Canada unifies medical research community
}

David Spurgeon, Montreal

A new era opened for Canadian medical researchers last week with the launch of the Canadian Institutes of Health Research (CIHR). The new body replaces the Medical Research Council of Canada, which has been the major federal agency funding health research and training since 1960 (see Nature 393, 613; 1998).

The CIHR will bear some resemblance to the US National Institutes of Health. But there are also important differences. Whereas the NIH has 25 institutes and centres housed in 75 buildings in Bethesda, Maryland, the CIHR will have no main campus. Its researchers will be grouped into 'virtual' institutes, located in 16 health-science centres, 50 teaching hospitals and 65 research institutes across the country.

The aim is to link investigators in biomedical and clinical research, health systems and services research, and populationhealth research across Canada. There will be no intramural research in the NIH sense: instead, work will be done by investigators in the participating organizations, although it will be evaluated and reviewed centrally by peer committees.

Among the advantages - apart from a

much bigger budget - is said to be the inclusion of a broader range of health research than any previous agency, encouraging closer collaboration between disciplines and possibly new avenues of research.

Alan Bernstein, former director of

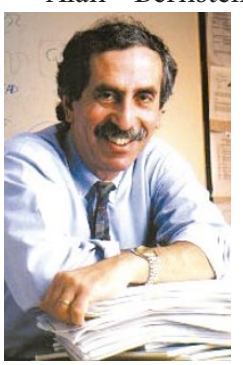

Bernstein: will preside over 'virtual' institutes. and formation of the cardiovascular system.

Biomedical researchers, although initially apprehensive, have welcomed the new arrangements. Cecil Yip, vice-dean of research at the University of Toronto, says that the CIHR is "a good thing from many perspectives, not the least of which is that it represents a serious commitment [to health research] from the federal government".
The budget of the institutes is set to double from the MRC's 1999-2000 figure of Can\$302.5 million (US\$184 million) over the next three years; it will be Can $\$ 402$ million in 2000-01, rising to Can $\$ 533$ million the following year. Henry Friesen, who was MRC president and chief architect of the CIHR, says that a budget of Can $\$ 1$ billion is both possible and defensible.

The creation of the CIHR was announced in the 1999 federal budget, following the report of a National Task Force on Health Research, made up of representatives from the whole medical research community.

A 19-member governing council will set the strategic direction, goals and policies of the component institutes - whose details have not yet been announced - and determine the mandate of each. It will also appoint the institutes' scientific directors and advisory boards.

Federal health minister Allan Rock says that the CIHR "represents an entirely new way of conducting health research ... its emphasis on partnerships with the voluntary healthcare sector, other government agencies and industry will be a model to be emulated around the world".

http://www.cihr.ca

\section{Miniature antennas will eavesdrop on the Universe}

\section{Steve Nadis}

Researchers at Ohio State University (OSU) are building a radio array, composed of 64 tiny antennas, as a prototype for telescopes that may revolutionize the search for extraterrestrial intelligence (SETI) and radioastronomy in general.

At roughly 100 square centimetres, each antenna is so small that its field of view extends from horizon to horizon, unlike dish-shaped antennas, which focus on a small portion of the sky. Eight antennas have been installed, with four already operating full-time. The team expects the entire array to be in place this autumn.

A telescope built this way could monitor the whole sky continuously over a wide range of radio frequencies. Not only might it pick up transient signals from extraterrestrial life, but it might also spot short-lived astrophysical phenomena missed by other instruments.

The project, funded by the Californiabased SETI Institute, has so far cost less than $\$ 200,000$. "We're working with a 64-element array because that's large enough to get into some of the technical issues, but small enough that we can put it together at a reasonable cost," says the principal investigator Steven Ellingson, an electrical engineer at OSU. "Our main goal is to figure out how to build and operate an entirely new kind of telescope."

One lesson that has already been learned, says Ellingson, is that, rather than trying to form images of the entire sky, “it's more efficient to use modern signal-processing techniques to figure out when and where something interesting is happening, and then focus on just that part of the sky".

The prototype is called Argus, after a Greek mythological hero with 100 eyes. The urban setting of Columbus, Ohio, is far from ideal for astronomy, which is why the array is intended primarily as an engineering test-bed, rather than a front-line observational tool.

Nevertheless, Argus can still make valuable contributions to radio astronomy. The instrument could, for instance, detect bright, ephemeral events such as supernovae and advise other observatories on where to point their telescopes.

$\mathrm{X}$-ray and gamma-ray bursts are another good example, says Frank Briggs, a radio astronomer at the University of Groningen in the Netherlands. "We can learn new things about these phenomena by looking at

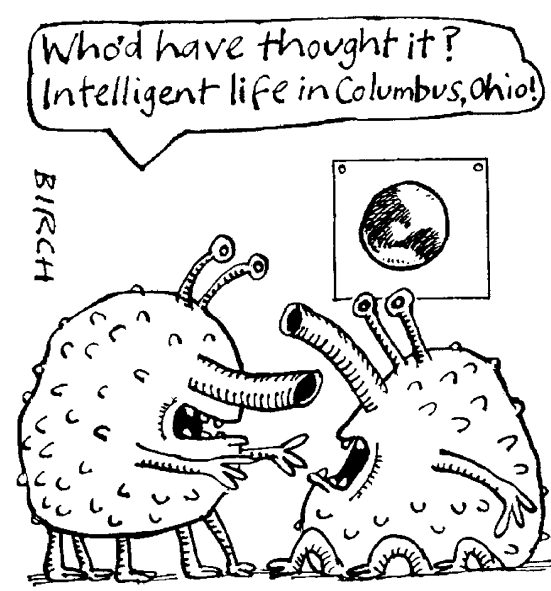

the radio signals they emit, as well as at the $\mathrm{X}$-rays and gamma rays."

Such an array could have tremendous potential, says Kent Cullers, a physicist at the SETI Institute - provided that costs can be kept down. The main expense for a larger, more sensitive instrument, perhaps linking a million antennas, would be the computing power tying them together, rather than the antennas themselves. "If computing costs keep coming down, this could well be the telescope of the future," says Cullers. 